Proceedings of the 33rd Annual Meeting of the Brazilian Embryo Technology Society (SBTE); Ilha de Comandatuba, BA, Brazil, August 15th to 19th, 2019.

\title{
Intensive use of IVF by large-scale dairy programs
}

\author{
Bruno Valente Sanches ${ }^{1}$, Amanda Fonseca Zangirolamo ${ }^{2,3}$, Marcelo Marcondes Seneda ${ }^{2,3, *}$ \\ ${ }^{1}$ Vytelle IVF, LLC, Hermiston, OR, USA. \\ ${ }^{2}$ Universidade Estadual de Londrina, Laboratório de Reprodução Animal, DCV-CCA-UEL, Londrina, Parana, Brazil. \\ ${ }^{3}$ National Institute of Science and Technology for Dairy Production Chain (INCT-LEITE), Universidade Estadual de Londrina, \\ Rodovia Celso Garcia Cid-Campus Universitário, Parana, Brazil.
}

\begin{abstract}
The number of embryos produced by in vitro fertilization (IVF) has grown exponentially in recent years. Recently, for the first time, the number of embryos produced and transferred in vitro was significantly higher than the number developed in vivo worldwide. In this context, a particular boost occurred with ovum pick-up (OPU) and in vitro embryos produced in North America, and this technology is becoming more prominent for commercial dairy farms. However, despite many advances in recent decades, laboratories and companies are looking for methods and alternatives that can be used in collaboration with the existing process to improve it. Among the strategies used to improve the dairy industry are the use of genomic analysis for the selection of animals with desired traits or as an evaluation tool of oocyte and embryo quality, the optimization of the collection and use of gametes from prepubertal females and males, the effective use of sexed semen, and improvements in culture media and methods of embryo cryopreservation. Thus, this review aims to discuss the highlights of the commercial use of IVF and some strategies to increase the application of this technique in large-scale dairy programs.
\end{abstract}

Keywords: IVF, bovine, dairy, commercial use, genomic analysis.

\section{Introduction}

The dairy industry plays an essential role in the global socioeconomic scenario. Although growth in global milk production has been limited in recent years, it is projected to increase by $22 \%$ in 2027 compared to 2015-2017 (OECD and FAO, 2018). The dairy industry is the leader among the food animal sector in the successful application of advanced technologies (Wiggans et al., 2017). Therefore, practices and alternatives that improve the production of dairy cattle are increasingly required.

The increase in the productive efficiency and quality of animal products from livestock has been possible due to the use of reproductive biotechniques (Hansen et al., 2014). In this context, in vitro fertilization (IVF) is a useful tool when performing the selection and breeding of genetically superior animals (Hansen et al., 2014), which is becoming more prominent in commercial dairies (Sirard, 2018).
According to the Embryo Transfer Newsletter (Viana, 2017), for the first time, the number of bovine embryos produced and transferred in vitro was significantly higher than those in vivo produced worldwide (Figs. 1-2; Viana, 2017).

Furthermore, for the first time since 1999, North America has reported more in vitro produced (IVP) embryos than South America, the region that led the use of IVF in the past decade (Fig. 3; Viana, 2018). Notably, the United States (US) had the highest number of IVP embryos within North America, at approximately 95.5\% (Viana, 2018). The further development of the embryo industry in North America seems to resemble what happened in South America, in which the contribution of in vivo embryos has been linear, and the use of IVP embryos has resulted in a substantial increase in numbers (Viana et al., 2018).

However, despite advances in IVF, the embryo production rate from the total cumulus oocyte complexes (COC), the embryo production rate remains at 30 to $40 \%$ (Lonergan et al., 2016). Thus, laboratories and companies have been looking for alternatives that collaborate to improve the existing process and to optimize methods to use IVF in large-scale dairy programs.

The genomic testing of cattle is now significantly affecting IVF programs. Genomic selection has revolutionized dairy farming, shortening the breeding interval, increasing selection accuracy, and reducing the previous costs of progeny testing (Wiggans et al., 2017). The commercial interest in performing genomic analysis and collecting gametes from prepubertal animals that have desired traits is increasing (Moore and Hasler, 2017). The small ultrasound OPU probes currently available allow IVP embryos from younger females to be grown (Sirard, 2018). Additionally, genomic analysis has been used to evaluate the quality and viability of oocytes, and even the embryos, before transfer procedures (Moore and Hasler, 2017).

An embryo culture media have been developed to mimic what happens in the maternal organism. Several studies have been performed to investigate the addition of different products and molecules in the culture medium, such as cytokines, growth factors, and antioxidants, and many advances have been obtained.

Sexing technology is another practice used to improve IVF results.; The use of sexed semen enables the birth of offspring of a predetermined sex, as well as increase the efficiency of producing donors with the right genetic background. Furthermore, IVF is the most 
common application of sexed semen, which has superior efficacy compared with its use in other areas of biotechnology (Morotti et al., 2014).

Due to the increasing number of IVP embryos, cryopreservation methods provide a good alternative for the storage of surplus products. However, some limitations exist, which may hamper the use of cryopreservation on a large scale. In this context, among the different protocols, the process of thawing and the direct transfer of embryos together make the cryopreservation protocol more efficient for commercial use by facilitating logistics in the field (Sanches et al., 2016).

Thus, this review aims to discuss some strategies to increase the useful application of IVF in large-scale dairy programs, as well as the trends, challenges, and highlights of the commercial use of the IVF program.

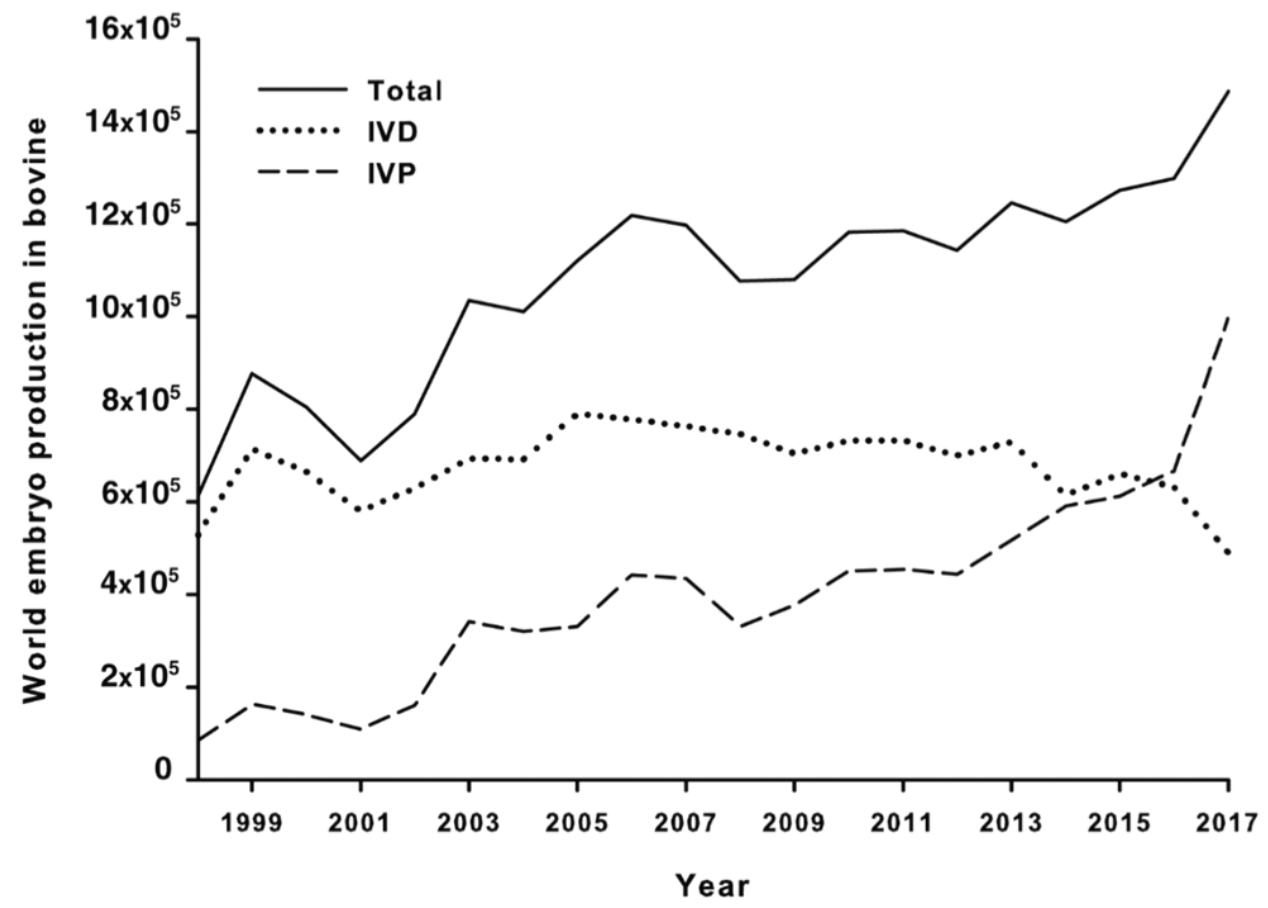

Figure 1. The number of bovine embryos produced (in vivo - IVD, in vitro - IVP, and total) recorded in the period 1998 - 2017 (Data sourced from Viana, 2017; Viana, 2018).

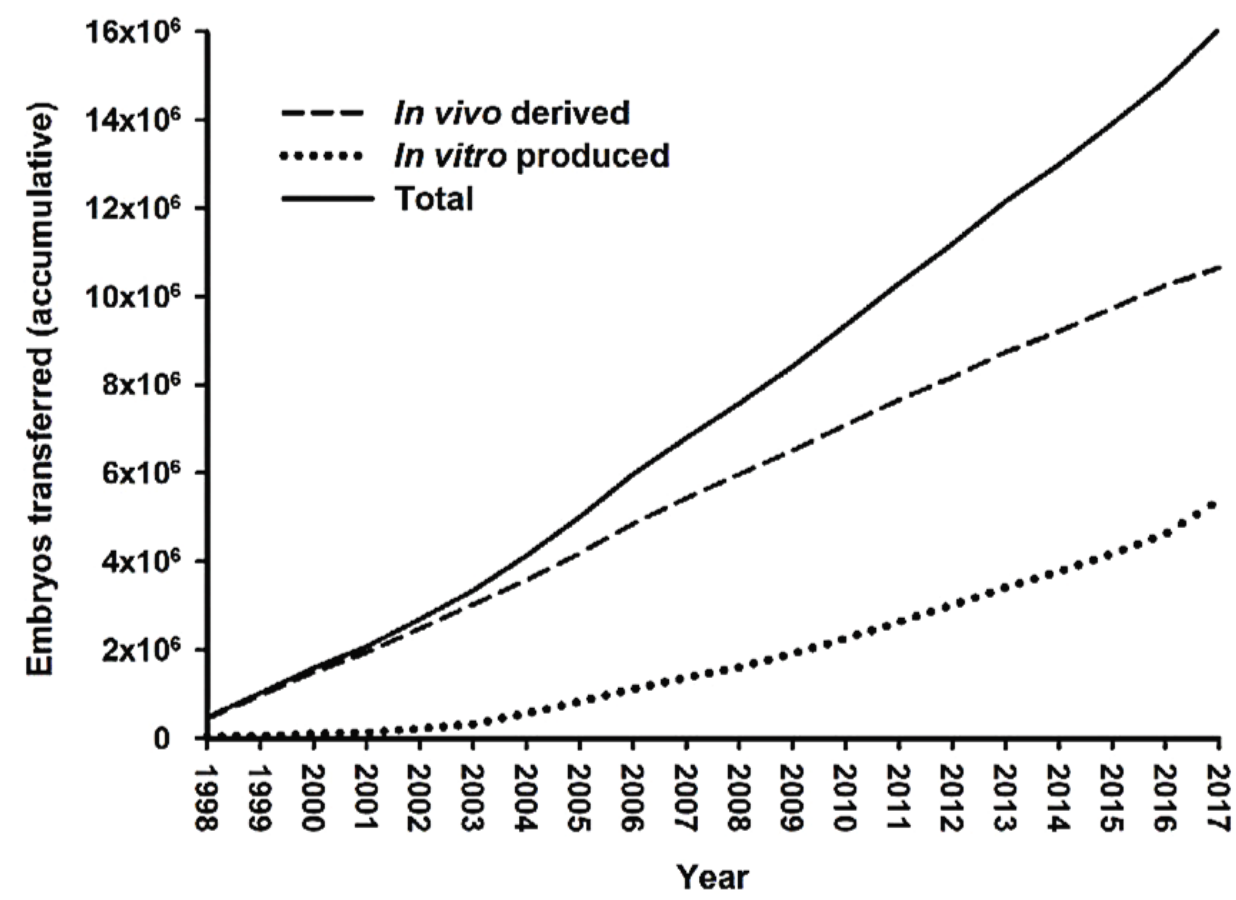

Figure 2. The accumulated number of bovine embryos transferred in the period 1998 - 2017, based on in vivo or in vitro production methods (Data sourced from Viana, 2017; Viana, 2018). 


\section{GLOBAL TRENDS IN IVP BY CONTINENT FROM 2013-17}

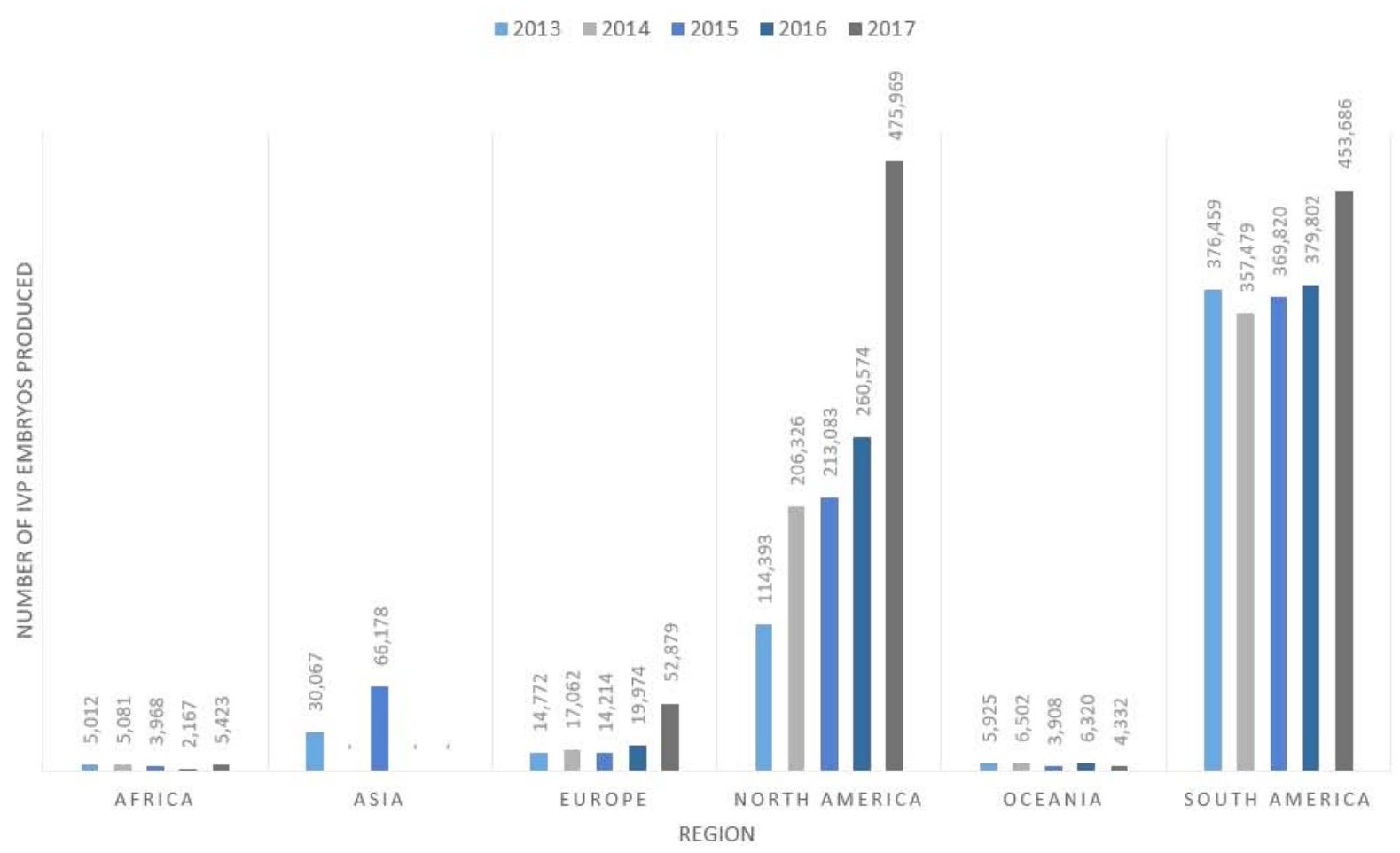

Figure 3. The accumulated number of IVP bovine embryos in the period 2013 - 2017 by continent (Data sourced from Viana, 2017; Viana, 2018).

\section{Genomic analysis: from animal selection to oocyte and embryo evaluation}

Currently, genomic analysis is driving the development of several IVF laboratories in North America and other places in the world (Sirard, 2018). The best effect of genomic selection to date has been to double the rate of genetic progress for traits of economic interest. Genetic improvement occurs through the increased accuracy of genetic merit for young animals (Wiggans et al., 2017).

With genomic analysis performed soon after birth, the genetic value of the bull is determined early, and as soon as semen is produced, such high genetic merit sperm, can be used for IVF. Moreover, it has increased the commercial demand for producing embryos from young heifers and calves (Sirard, 2018). Currently, the collection of oocytes from donors before puberty is possible with relatively high success (Landry et al., 2016). Additionally, genomic selection is helpful for choosing better embryo recipients according to the genes involved with gestation maintenance.

Genomic evaluations for Holsteins, Jerseys, and Brown Swiss became official in 2009 at the USDA, and since that time, more than 1 million animal genotypes have received genetic evaluations (Council on Dairy Cattle Breeding, 2016). Due to the popularity of genotyping chips, microsatellites have been replaced by SNPs, and the accessibility to chips of lower cost has made whole herd genotyping common in the US (Wiggans et al., 2017). The scenario of genotyped animals included in US genomic evaluations for dairy cattle is shown in Fig. 4.

Additionally, dairy cattle can be selected for any combination of traits, but total genetic progress will be fastest using an index because many traits affect profitability. In this context, the lifetime net merit (NM\$) index, elaborated by the U.S. Department of Agriculture (USDA), ranks dairy animals based on their combined genetic merit for economically important traits (VanRaden, 2018). The NM\$ index includes economically important traits related to health, yield, longevity and calving ease, and because it is calculated using Holstein values, it is, therefore, widely used for this breed (VanRaden, 2018). The weighting and composition of the 14 traits that make up the net merit in the year 2018 are shown in Fig. 5.

However, the relative importance of traits differed slightly between the production systems. Organic dairy producers, for example, tend to prefer health traits as the pillar of selection, even though the increase in the genetic gain in disease resistance is achieved at the expense of milk production, since they cannot use any medicine or chemical in animals (Fall et al., 2008). Moreover, the CM\$ provides longevity and somatic cell score data for producers whose milk is made into cheese or other dairy products (VanRaden, 2017). Although the Jersey produces less milk than the Holstein, it produces milk with more fat, milk protein, and a higher energy content (Aikman, et al., 2007). Therefore, for Jersey cows, the CM\$ would possibly be more interesting.

Another application of genomic analysis is the use of micro-array or RNAseq technologies on embryos 
submitted to different culture conditions, with the intention of comparing in vivo control embryos and, thus, improving culture methods (Sirard, 2018). Furthermore, embryo culture medium can provide a source of material for noninvasive embryonic genetic testing (without biopsies, for example). However, whether the DNA found represents the genetic state of the embryo remains unknown. Thus, this potentially noninvasive approach must be validated by additional experiments (Liu et al., 2017; Smith et al., 2019), and once confirmed, it can lead to other methods to evaluate the quality of embryos.

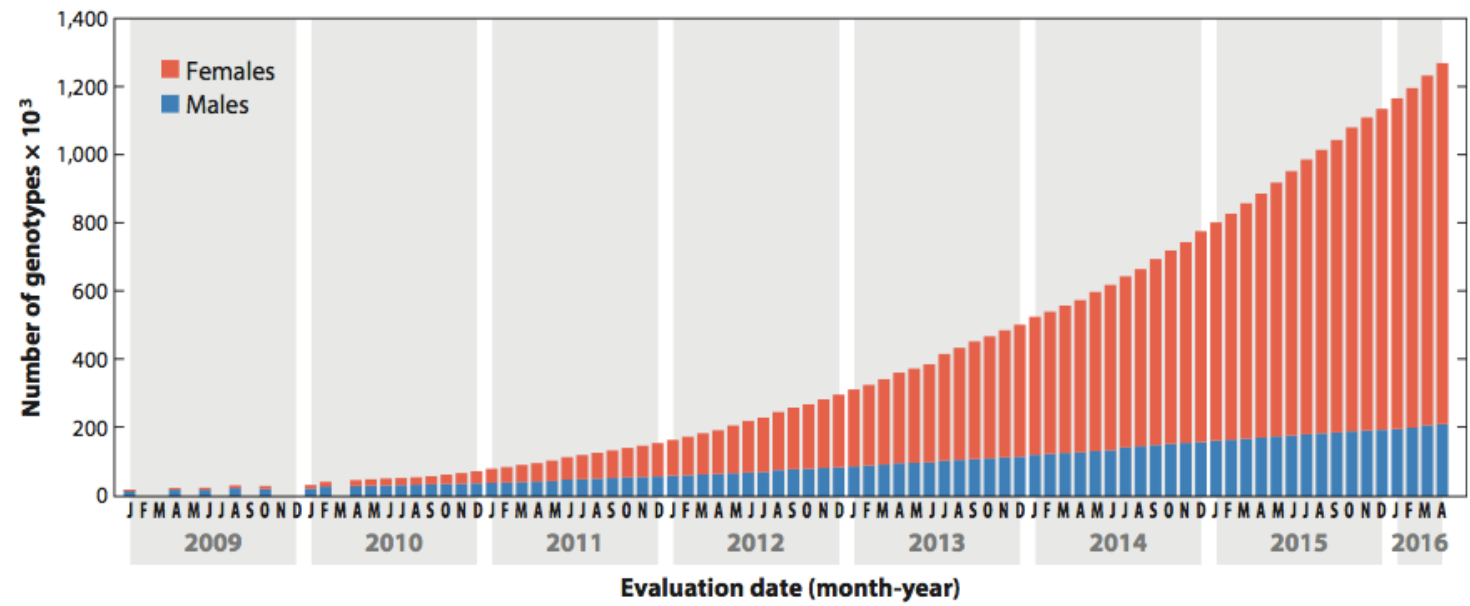

Figure 4. The number of genotyped animals included in US genomic evaluations for dairy cattle since January 2009 (Data sourced from the Council on Dairy Cattle Breeding, 2016; Wiggans et al., 2017).

\section{WEIGHTING OF TRAITS IN 2018 NM\$ (HOLSTEINS)}

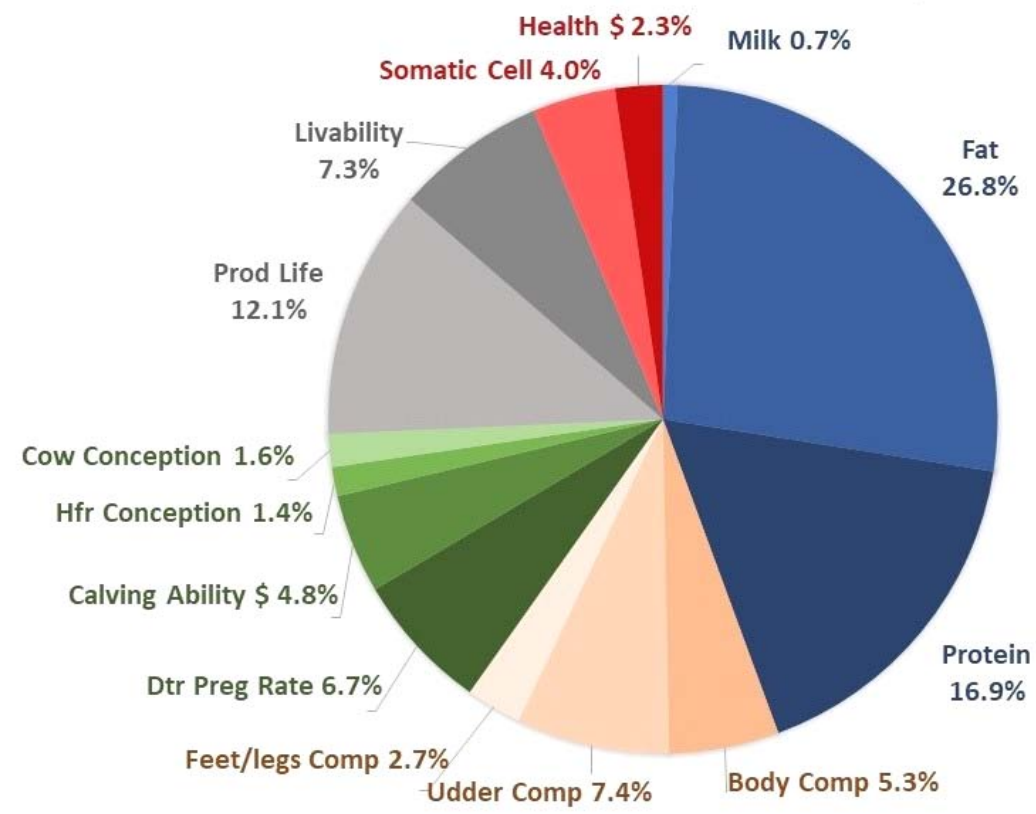

Figure 5. Composition and weighting of the 14 traits in 2018 Net Merit. (Available on: https://hoards.com/article23717-net-merit-\$-index-updated-to-include-health-traits.html. Accessed on April 10, 2019).

\section{Strategies to improve oocyte competence before OPU}

The quality of the oocyte is the central factor interfering with the blastocyst yield, as well as the potential explanation for the limited success rates of IVF (Lonergan and Fair, 2016). The oocyte competence, and consequently the development to the blastocyst stage, is positively associated with the size of the antral follicle (Lonergan et al., 1994) and whether the blastocyst was produced in vivo or in vitro (Rizos et al., 2002).

One strategy for manipulating follicular growth and affecting developmental competence is Coasting, which is a period between hormonal stimulation and ovary collection (Nivet et al., 2012). In adult females, this approach allowed a high rate of blastocyst development after IVF, suggesting an increase in oocyte quality (Blondin et al., 2002). Animals that received six 
injections of FSH, followed by a 48-h coasting period and an injection of LH 6 h before ovum pick-up (OPU), presented an $80 \%$ rate of blastocyst stage occurrence (Blondin et al., 2002). More recently, the same group showed that the ideal period was $54 \pm 7$ hours, where a well-defined period of competence to recover oocytes of the highest quality is of paramount importance (Nivet et al., 2012).

During OPU oocytes are recovered on random days of the estrous cycle, i.e., follicles that are at different stages of development (Wit et al., 2000). In these conditions, more than $85 \%$ of the aspirated ovarian follicles present some degree of atresia due to the apoptosis process (Hendriksen et al., 2000). Recently, follicular wave synchronization before OPU was observed to provide an increase in embryo production rates and post-transfer conception for the recipients (Cavalieri et al., 2018).

\section{Collection and quality of oocytes from prepubertal heifers and calves}

Recently, there has been an increase in the commercial interest in producing bovine embryos from prepubertal heifers and calves. The interest in breeding the best animals at younger ages is to accelerate the genetic advancement rate of genetic gain (Baldassarre $e t$ al., 2018). With the emergence of genomic technologies in recent years, the prediction of better phenotype production has been possible after birth of the animal (Ponsart et al., 2013).

In the early 1990s, the development of transvaginal oocyte recovery procedures in cattle improve the IVF method (Pieterse et al., 1991). Initially, due to animal size issues, the collection of oocytes by OPU was very difficult or not possible, and laparoscopic ovum pick-up (LOPU) rapidly became the method of choice for small animals such as calves and pre-pubertal heifers (Cognie et al., 2004). Currently, small ultrasound OPU probes are available and allow IVP embryos from younger females to be grown (Moore and Hasler, 2017).

Several studies have shown that bovine calf oocytes are significantly less capable of developing into embryos compared with oocytes from adult cows (Baldassarre and Bordignon, 2018). Prepubertal females have immature and nonfunctional hypothalamuspituitary-ovarian axes and, therefore, are unable to achieve full follicular development and ovulation (Sanchez and Smitz, 2012). Thus, different research groups are directing efforts seeking to improve quality and increase oocyte competence in young heifers.

Studies described the recovery of a high number of oocytes from females 2-6 months of age who were stimulated with gonadotropins to increase the proportion (and size) of large follicles (Baldassarre and Bordignon, 2018). In some cases, the number of oocytes was higher than what was recovered from adult cows.

$B$. taurus and $B$. indicus aging from 2 to 4 months did not exhibit an improvement in IVF results when stimulated with $140 \mathrm{mg}$ of FSH (Batista et al., 2016). However, recently, more prolonged FSH stimulation (three days) was shown to increase the development competence of Holstein calf oocytes, which was associated with a higher proportion of follicles larger than $5 \mathrm{~mm}$ (Currin et al., 2017).

A previous study showed that Holstein calves aging from 5 to 7 months had more oocytes than cycling heifers aging from 16 to 18 months. Although the blastocyst rate was higher in the cycling heifers than in the calves, the number of embryos (6-8) was not different (Landry et al., 2016). Further studies are necessary to investigate the beneficial effects of exogenous gonadotropins to prepubertal heifers and calves.

Other possible future efforts of research include the development of in vitro maturation (IVM) protocols with strategies for delaying nuclear and improving cytoplasmic maturation. Additionally, another research target includes the supplementation of IVM medium with substances or molecules that improve oocyte development for step embryo transfer (Baldassarre and Bordignon, 2018). We also believe that the epigenetic changes or even the nutrition of the mother, which can interfere in the quality of the oocytes of the daughters, will be an area for future research.

Thus, with the advent of genomic analysis, the extraordinary growth of IVF technologies in recent years and high interest by dairy producers and the use of elite females that are as young as possible (from 2 months of age) for embryo production has the potential to help IVF become a viable practice very soon.

\section{Advances in embryo culture media}

For in vitro embryo production (IVEP), specific media are used for maturation, fertilization, and in vitro culture to mimic what occurs physiologically in the organism. In cattle, approximately $90 \%$ of immature oocytes, recovered from follicles at unknown stages of the estrous cycle (ovaries from slaughterhouse), undergo nuclear maturation in vitro and approximately 80\% undergo fertilization (Lonergan and Fair, 2016).

The media used may be a determinant factor in the production and quality of blastocysts and embryo cryotolerance (Sanches et al., 2013). Changes in the culture conditions such as the addition of lipolytic chemical substances and the adjustment of fetal calf serum in the medium have been proposed to increase the embryo cryotolerance (Sanches et al., 2017). In this context, several studies show forskolin and phenazine ethosulfate (PES), as substances which reduces lipid accumulation (Sudano et al., 2011; Paschoal et al., 2017).

Although modest improvements have occurred in the development and composition of IVM media (addition of different products, cytokines, growth factors, antioxidants, and other substances), the blastocyst rate rarely exceeds 40-50\% (Lonergan and Fair, 2016). Thus, the yield of oocytes developing to the blastocyst stage remains very similar to that in the years 1990 to 2000, in which it reached a plateau at 30-40\% (Sirard, 2018). 
Another strategy to improve embryo culture media is to try to keep what occurs physiologically in the follicular environment of the oocyte, in which the arrest of meiosis is maintained. Several meiotic inhibitors can delay the resumption of in vitro meiosis. Thereby, the continuous accumulation of mRNA and proteins within the oocyte allows a better cytoplasmic maturation (Bilodeau-Goessels, 2012).

However, despite the many protocols and tested methods in vitro, attention has turned toward the source of oocytes as the cause of the limited success rates of IVF (Sirard, 2018).

\section{Use of sexed semen and its advantages}

In the dairy industry, the production of overweight calves from undesirable sex (i.e. male) is a particularly important issue (Holden and Butler, 2018) The use of sexed semen in association with reproductive biotechnologies represents a significant advance in the global livestock industry. In this context, the predetermination of the sex of the animal optimizes production and profitability in dairy herds (Morotti et al., 2014).

Among the reproductive biotechnologies, the most common application of sexed semen is IVF due to good blastocyst rates can be achieved (Matoba et al., 2014). Moreover, IVF to require far less sperm per oocyte to make acceptable fertilization rates compared with AI (Holden and Butler, 2018). In the US, more than $90 \%$ of 4.5 to 5 million straws of sexed semen were from milk dairy bull sires in 2016 (Moore and Hasler, 2017).

However, studies show that the blastocyst rates are lower than those obtained with conventional semen (Seidel Jr. 2014). On the other hand, Cottle et al. (2018) identified a significant profit advantage for using sexed semen in the context of a high-output, dairy system of spring births in Ireland. The authors concluded that the use of sexed semen is more appropriate for those farms that already have an excellent fertility performance. Thus, the lowest rates associated with sexed semen can be less acceptable for farms with sub-optimal dairy herds fertility (Cottle et al., 2018).

In any case, genetic targeting of the dairy herd to achieve desired sex animals justifies the expansion of the use of sexed semen in the dairy sector.

\section{Advances in cryopreservation with the use of DT}

Despite the IVF advantages, cryopreservation represents a challenge for commercial laboratories. The low cryotolerance of IVP embryos is a limiting factor to the use of the cryopreservation process in an IVF program (Sudano et al., 2011). In addition, after the cryopreservation is well established, we believe the number of field technicians trained to do the transfer process will not be sufficient. Thus, efforts should be made to overcome all limitations involving the use of IVP embryo cryopreservation on a large scale and globally.

Among cryopreservation techniques, vitrification is more often used worldwide due to the speed and low cost (Dode, 2013). However, its method requires a high concentration of cryoprotectants and a trained professional to evaluate embryo quality prior to the transfer (Vajta and Kuwayama, 2006).

In contrast, the direct transfer (DT), a method used since the 1990s to simplify the post-thawing rehydration step of in vivo embryos, has been proving to be a useful alternative for commercial use in IVP embryos.

In a study with Girolando donors (1/2 Gir and $1 / 2$ Holstein), the conception rates obtained were 51.35 $\pm 1.87 \%(133 / 259)$ for the fresh embryos, $35.89 \pm$ $3.87 \%(84 / 234)$ for the vitrified embryos, and $40.19 \pm$ $4.65 \%$ (125/311) for the embryos submitted to DT (Sanches et al., 2016). Possibly, IVP embryos with sexed semen could be directly transferred with similar conception rates to those submitted to vitrification.

The low concentration of cryoprotectants is the main advantage of this technique because of the reduced toxicity to the embryos (Voelkel and $\mathrm{Hu}, 1992$ ). Furthermore, the DT eliminates the evaluation before transfer and, therefore, is more practical than vitrification (Sanches et al., 2017). Finally, due to the promising results, DT has been implemented in largescale operations, mainly in the US and Brazil.

\section{Final comments}

The genomic selection of young animals, associated with sexed semen and frozen IVP-blastocysts and following direct transfer protocols, is driving a new era of IVF in the dairy sector (Sirard, 2018). However, since many of these processes are sensitive to operators or even the environment, the challenge of making IVF fully business-grade remains.

In the US, some large dairies have already left behind the commercially available genomic tests and have begun to implement their own genomic assessments and methods for identifying the best animals to be reproduced. Therefore, this behavior of the industry indicates that companies in the business of IVF also need to invest in innovation to develop a more personalized product because, ultimately, they must go beyond the goal of delivering a quality embryo and/or ensuring a high pregnancy rate.

\section{Author contributions}

AFZ: Conceptualization, Writing, Data curation; BVS: Conceptualization, Editing; MMS: Supervision.

\section{Conflict of interest}

Authors declare there is no conflict of interest.

Acknowledgments and Funding disclosure statement

MMS: supported by CNPq 403862/2016-7 and CNPq 308460/2018-9. National Institute of Science and Technology for Dairy Production Chain. 


\section{References}

Aikman PC, Reynolds CK, Beever DE. 2007. Diet digestibility, rate of passage, and eating and rumination behavior of jersey and holstein cows. J Dairy Sci, 91:1103-1114.

Baldassarre H, Bordignon V. 2018. Laparoscopic ovum pick-up for in vitro embryo production from dairy bovine and buffalo calves. Anim Reprod, 15:191-196.

Baldassarre H, Currin L, Michalovic L, Bellefleur A, Gutierrez K, Mondadori RG, Glanzner WG, Schuermann Y, Bohrer RC, Dicks N, Lopez R, Grand FX, Vigneault C, Blondin P, Gourdon J, Bordignon V. 2018. The interval of gonadotropin administration for in vitro embryo production from oocytes collected from Holstein calves between 2 and 6 months of age by repeated laparoscopy. Theriogenology, 116:64-70.

Batista EOS, Guerreiro BM, Freitas BG, Silva JCB, Vieira LM, Ferreira RM, Rezende RG, Basso AC, Lopes RNVR, Rennó FP, Souza AH, Baruselli PS. 2016. Plasma anti-Müllerian hormone as a predictive endocrine marker to select Bos taurus (Holstein) and Bos indicus (Nelore) calves for in vitro embryo production. Domest Anim Endocrinol, 54:1-9.

Bilodeau-Goeseels S. 2012. Bovine oocyte meiotic inhibition before in vitro maturation and its value to in vitro embryo production: Does it improve developmental competence? Reprod Domest Anim, 47:687-93.

Blondin P, Bousquet D, Twagiramungu H, Barnes F, Sirard MA. 2002. Manipulation of follicular development to produce developmentally competent bovine oocytes. Biol Reprod, 66:38-43.

Cavalieri FLB, Morotti F, Seneda MM, Colombo AHB, Andreazzi MA, Emanuelli IP, Rigolon LP. 2018. Improvement of bovine in vitro embryo production by ovarian follicular wave synchronization prior to ovum pick-up. Theriogenology, 57:70-117.

Cognie Y, Poulin N, Locatelli Y, Mermillod P. 2004. State-of-the-art production, conservation and transfer of in-vitro-produced embryos in small ruminants. Reprod Fertil Dev, 16:437-445.

Cottle DJ, Wallace M, Lonergan P, Fahey AG. 2018. Bioeconomics of sexed semen utilization in a highproducing Holstein-Friesian dairy herd. J Dairy Sci, 101: 4498-4512.

Council on Dairy Cattle Breeding. 2016. Genotypes Included in Evaluations by Breed, Chip Density, Presence of Phenotypes (Old vs. Young), and Evaluation Year-Month (Cumulative). Bowie, MD: Council Dairy Cattle Breed. https://www.cdcb.us/ Genotype/cur_density.html.

Currin L, Michalovic L, Bellefleur AM, Gutierrez K, Glanzner W, Schuermann Y, Bohrer RC, Dicks N, da Rosa PR, De Cesaro MP, Lopez R, Grand FX, Vigneault C, Blondin P, Gourdon J, Baldassarre H, Bordignon V. 2017. The effect of age and length of gonadotropin stimulation on the in vitro embryo development of Holstein calf oocytes. Theriogenology, 104:87-93.
Dode MAN, Leme LO, Spricigo JFW. 2013. Criopreservação de embriões bovinos produzidos in vitro. Rev Bras Reprod Anim, 37:145-150.

Fall N, Forslund K, Emanuelson U. 2008. Reproductive performance, general health, and longevity of dairy cows at a Swedish research farm with both organic and conventional production. Livest Sci, 118:11-19.

Hansen P. 2014. Current and Future Assisted Reproductive Technologies for Mammalian Farm Animals. Adv Exp Med Biol, Advances in Experimental Medicine and Biology, 752:1-22.

Hendriksen PJ, Vos PL, STeenweg WN, Bevers MM, DIeleman SJ. 2000. Bovine follicular development and its effect on the in vitro competence of oocytes. Theriogenology, 53:11-20.

Holden SA, Butler ST. 2018. Review: Applications and benefits of sexed semen in dairy and beef herds. Animal, 12(s1):s97-s103

Landry DA, Bellefleur AM, Labrecque R, Grand FX, Vi- gneault C, Blondin P, Sirard MA. 2016. Effect of cow age on the in vitro developmental competence of oocytes obtained after FSH stimulation and coasting treatments. Theriogenology, 86:1240-1246. Liu W, Liu J, Du H, Ling J, Sun X, Chen D. 2017. Non-invasive preimplantation aneuploidy screening and diagnosis of beta thalassemiaIVSII654 mutation using spent embryo culture medium. Ann Med, 49:319-328.

Lonergan P, Monaghan P, Rizos D, Boland MP, Gordon I. 1994. Effect of follicle size on bovine oocyte quality and developmental competence following maturation, fertilization, and culture in vitro. Mol Reprod Dev, 37:48-53

Lonergan P, Fair T. 2016. Maturation of Oocytes in vitro. Annu Rev Anim Biosci, 4:10.1-10.14.

Lonergan P, Fair T, Forde N, Rizos D. 2016. Embryo development in dairy cattle. Theriogenology, 86:270-277. Matoba S, Yoshioka H, Matsuda H, Sugimura S, Aikawa Y, Ohtake M, Hashiyada $Y$, Seta $T$, Nakagawa K, Lonergan P. 2014. Optimizing production of in vivo-matured oocytes from superstimulated Holstein cows for in vitro production of embryos using X-sorted sperm. J Dairy Sci, 97:743-753. Moore SG, Hasler JF. 2017. A 100-Year Review: Reproductive technologies in dairy science. J Dairy Sci, 100:10314-10331.

Morotti F, Sanches B, Pontes J, Basso A, Siqueira E, Lisboa L, Seneda M. 2014. Pregnancy rate and birth rate of calves from a large-scale IVF program using reverse-sorted semen in Bos indicus, Bos indicus-taurus, and Bos taurus cattle. Theriogenology, 81:696-701.

Nivet A-L, Bunel A, Labrecque $R$, Belanger $J$, Vigneault C, Blondin P, Sirard M.A. 2012. FSH withdrawal improves developmental competence of oocytes in the bovine model. Reproduction, 143:165-171.

Organisation for Economic Co-operation and Development (OECD), Food and Agriculture Organization (FAO). 2018. Agricultural Outlook 2018-2027: Dairy and dairy products. 2018:29pp.

Paschoal DM, Sudano MJ, Schwarz KRL, Maziero RRD, Guastali MD, Crocomo LF, Magalhães LCO, 
Martins Jr, A, Leal ALV, Landim-Alvarenga FC. 2017. Cell apoptosis and lipid content of in vitroproduced, vitrified bovine embryos treated with forskolin. Theriogenology, 87:108-114.

Pieterse MC, Vos PL, Kruip TA, Wurth YA, van Beneden TH, Willemse AH, Taverne MA. 1991 Transvaginal ultrasound guided follicular aspiration of bovine oocytes. Theriogenology, 35:857-862.

Ponsart C, Le BD, Knijn H, Fritz S, Guyader-Joly C, Otter T, Lacaze S, Charreaux F, Schibler L, Dupassieux D, Mullaart E. 2013. Reproductive technologies and genomic selection in dairy cattle. Reprod Fertil Dev, 26:12-21.

Rizos D, Ward F, Duffy P, Boland MP, Lonergan P 2002. Consequences of bovine oocyte maturation, fertilization or early embryo development in vitro versus in vivo: implications for blastocyst yield and blastocyst quality. Mol Reprod Dev, 61:234-48.

Sanches BV, Marinho LSR, Filho BDO, Pontes JHF, Basso AC, Meirinhos MLG, Silva-Santos KC, Ferreira CR, Seneda MM. 2013. Cryosurvival and pregnancy rates after exposure of IVF-derived Bos indicus embryos to 17 forskolin before vitrification. Theriogenology, 80:372-377.

Sanches BV, Lunardelli PA, Tannura JH, Cardoso BL, Pereira MHC, Gaitkoski G, Basso AC, Arnold DR, Seneda MM. 2016. A new direct transfer protocol for cryopreserved IVF embryos. Theriogenology, 85:1147-1151.

Sanches BV, Zangirolamo AF, Silva NC, Morotti F, Seneda MM. 2017. Cryopreservation of in vitroproduced embryos: challenges for commercial implementation. Anim Reprod, 14:521-527.

Sanchez F, Smitz J. 2012. Molecular control of oogenesis. Biochim Biophys Acta, 1822:1896-1912.

Seidel Jr, GE. 2014. Update on sexed semen technology in cattle. Animal, 8:160-164.
Smith HL, Stevens A, Minogue B, Sneddon S, Shaw L, Wood L, Adeniyi T, Xiao H, Lio P, Kimber SJ, Brison DR. 2019. Systems based analysis of human embryos and gene networks involved in cell lineage allocation. BMC Genomics, 20:171.

Sirard M. 2018. 40 years of bovine IVF in the new genomic selection context. Reproduction, 156:R1-R7.

Sudano MJ, Paschoal DM, Rascado TD, Magalhaes LCO, Crocomo LF, Lima-Neto JF, LandimAlvarenga FD. 2011. Lipid content and apoptosis of in vitro-produced bovine embryos as determinants of susceptibility to vitrification. Theriogenology, 75:12111220.

Vajta G, Kuwayama M. 2006. Improving cryopreservation systems. Theriogenology, 65:236-244.

VanRaden PM. 2017. Net merit as a measure of lifetime profit: 2017 revision. AIP Research Report, NM\$6:2-17.

VanRaden PM. 2018. Net merit as a measure of lifetime profit: 2018 revision. AIP Research Report, 7:5-18.

Viana JHM. 2017. Statistics of embryo collection and transfer in domestic farm animals. Embryo Transfer. Newsletter, 36:08-25.

Viana JHM, Figueiredo ACV, Gonçalves RLR, Siqueira LGB. 2018. A historical perspective of embryo-related technologies in South America. Anim Reprod, 15:963- 970.

Voelkel SA, Hu YX. 1992. Direct transfer of frozenthawed bovine embryos. Theriogenology, 37:23-37.

Wiggans GR, Cole JB, Hubbard SM, Sonstegard TS. 2017. Genomic Selection in Dairy Cattle: The USDA Experience. Ann Rev Anim Biosci, 5:13.1-13.19.

Wit AAC, Wurth YA, Kruip AM. 2000. Effect of ovarian and follicle quality on morphology and developmental capacity of the bovine cumulus-oocyte complex. J Anim Sci, 78:1277-1283. 